Chirurg 2021 -92:959

https://doi.org/10.1007/s00104-021-01503-9

Angenommen: 11. August 2021

Online publiziert: 14 . September 2021

(c) Springer Medizin Verlag $\mathrm{GmbH}$, ein Teil von Springer Nature 2021

\section{Intermittierende pneumatische Kompression als Ergänzung zur postoperativen Thromboseprophylaxe für Hochrisikopatienten}

\author{
M. Schrempf $\cdot$ M. Anthuber \\ Klinik für Allgemein- , Viszeral- und Transplantationschirurgie, Universitätsklinikum Augsburg, Augsburg, \\ Deutschland
}

\section{Originalpublikation}

Lobastov K, Saaintu E, Aaceehlnv E et al (2021) Intermittent pneumatic compression in addition to standard prophylaxis of postoperative venous thromboembolism in extremely high-risk patients (IPC SUPER): A randomized controlled trial. Ann Surg 274:63-69

Hintergrund. Ausgedehnte abdominelle Operationen erhöhen das Risiko für postoperative thromboembolische Komplikationen wie tiefe Venenthrombosen (DVT) und Lungenembolien. Als Alternative zur Thromboseprophylaxe mit niedermolekularem Heparin (NMH) wird bei Patienten mit erhöhtem Blutungsrisiko in ausgewählten Fällen eine intermittierende pneumatische Kompression (IPK) eingesetzt. Die Kombination beider Modalitäten kann eine zusätzliche Reduktion des Thromboserisikos ohne Erhöhung des Blutungsrisikos bewirken und ist daher insbesondere für Patienten mit einem erhöhten Thromboserisiko interessant [3]. Eine russische randomisierte Studie hat den perioperativen kombinierten Einsatz beider Modalitäten bei Hochrisikopatienten untersucht.

Methoden. Die Studie wurde an zwei Zentren zwischen Februar 2017 und Oktober 2018 durchgeführt. Patienten mit einer geplanten abdominellen, retroperitonealen, thorakalen oder intrakraniellen Operation miteinem Caprini-Score $\geq 11$ [2] und einem Mindestalter von 40 Jahren wurden in die
Studie eingeschlossen. Je nach Randomisierung erhielten die Patienten entweder eine Thromboseprophylaxe mittels NMH (Kontrollgruppe) oder mittels NMH und IPK (IPK-Gruppe). Primärer Endpunkt war das Auftreten einer DVT, nachgewiesen mittels Sonographie, die alle 3 bis 5 Tage bis zur Entlassung durchgeführt wurde.

Ergebnisse. Insgesamt 407 Patienten wurden in die Studie eingeschlossen. Eine DVT trat bei $0,5 \%(n=1)$ der Patienten in der IPK-Gruppe und 16,7\% $(n=34)$ der Patienten in der Kontrollgruppe auf (RR 0,03; 95\%-Konfidenzintervall [KI] $0,01-0,21 ; p<0,001)$. Die Anzahl an Lungenembolien unterschied sich zwischen beiden Gruppen nicht signifikant (0\% vs. 2,5\%; RR 0,09; $95 \%-K I$ 0,01-1,63). Intention-to-treat- und Per-protocol-Analysen unterschieden sich nicht hinsichtlich des primären Endpunkts. In der Subgruppenanalyse zeigte sich der größte Vorteil einer zusätzlichen IPK für Patienten mit malignen Erkrankungen, die eine ausgedehnte abdominelle Operation erhielten.

\section{Kommentar}

Individuelle Risikofaktoren, wie ein erhöhter BMI (Body-Mass-Index), hohes Alter, Tumorerkrankungen, Immobilisation und bestimmte genetische Prädispositionen, beeinflussen das individuelle Thromboserisiko und werden in der vorliegenden Studie durch die Anwendung des Caprini-Scores bei der Selektion von Patienten berück- sichtigt. Dies könnte erklären, warum sich in der vorliegenden Arbeit im Gegensatz zur PREVENT-Studie ein eindeutiger Vorteil für die zusätzliche Anwendung einer IPK zeigt. Diese untersuchte den zusätzlichen Einsatz der IPK bei intensivpflichtigen Patienten und konnte keinen Vorteil für die IPK nachweisen [1]. Angesichts der Ergebnisse erscheint ein routinemäßiges Aufnahmescreening mittels Caprini-Score in der Allgemein- und Viszeralchirurgie sinnvoll, um genau die Patienten zu selektieren, die von einer Kombinationstherapie profitieren.

\section{Korrespondenzadresse}

Prof. Dr. M. Anthuber

Klinik für Allgemein- , Viszeral- und

Transplantationschirurgie, Universitätsklinikum Augsburg

Stenglinstr. 2, 86156 Augsburg, Deutschland matthias.anthuber@uk-augsburg.de

Interessenkonflikt. M. Schrempf und M. Anthuber geben an, dass kein Interessenkonflikt besteht.

\section{Literatur}

1. Arabi YM, Al-Hameed F, Burns KEA et al (2019) Adjunctive intermittent pneumatic compression for venous thromboprophylaxis. N Engl J Med 380:1305-1315

2. Caprini JA, Arcelus Jl, Hasty JH et al (1991) Clinical assessment of venous thromboembolic risk in surgical patients. Semin Thromb Hemost 17(Suppl 3):304-312

3. Kakkos SK, Caprini JA, Geroulakos G et al (2016) Combined intermittent pneumatic leg compression and pharmacological prophylaxis for prevention of venous thromboembolism. Cochrane Database Syst Rev 9:CD5258 